\title{
Assembly, annotation, and comparison of Macrophomina phaseolina isolates from strawberry and other hosts
}

\author{
Alyssa K. Burkhardt ${ }^{1 *}$ D, Kevin L. Childs ${ }^{2 *}$, Jie Wang², Marina L. Ramon and Frank N. Martin ${ }^{1 *}$
}

\begin{abstract}
Background: Macrophomina phaseolina is a fungal plant pathogen with a broad host range, but one genotype was shown to exhibit host preference/specificity on strawberry. This pathogen lacked a high-quality genome assembly and annotation, and little was known about genomic differences among isolates from different hosts.

Results: We used PacBio sequencing and $\mathrm{Hi}-\mathrm{C}$ scaffolding to provide nearly complete genome assemblies for $\mathrm{M}$. phaseolina isolates representing the strawberry-specific genotype and another genotype recovered from alfalfa. The strawberry isolate had 59 contigs/scaffolds with an N50 of $4.3 \mathrm{Mb}$. The isolate from alfalfa had an N50 of $5.0 \mathrm{Mb}$ and 14 nuclear contigs with half including telomeres. Both genomes were annotated with MAKER using transcript evidence generated in this study with over 13,000 protein-coding genes predicted. Unique groups of genes for each isolate were identified when compared to closely related fungal species. Structural comparisons between the isolates reveal large-scale rearrangements including chromosomal inversions and translocations. To include isolates representing a range of pathogen genotypes, an additional 30 isolates were sequenced with Illumina, assembled, and compared to the strawberry genotype assembly. Within the limits of comparing Illumina and PacBio assemblies, no conserved structural rearrangements were identified among the isolates from the strawberry genotype compared to those from other hosts, but some candidate genes were identified that were largely present in isolates of the strawberry genotype and absent in other genotypes.

Conclusions: High-quality reference genomes of $M$. phaseolina have allowed for the identification of structural changes associated with a genotype that has a host preference toward strawberry and will enable future comparative genomics studies. Having more complete assemblies allows for structural rearrangements to be more fully assessed and ensures a greater representation of all the genes. Work with Illumina data from additional isolates suggests that some genes are predominately present in isolates of the strawberry genotype, but additional work is needed to confirm the role of these genes in pathogenesis. Additional work is also needed to complete the scaffolding of smaller contigs identified in the strawberry genotype assembly and to determine if unique genes in the strawberry genotype play a role in pathogenicity.
\end{abstract}

Keywords: Macrophomina phaseolina, Genome assembly, Genome annotation, Strawberry, Host preference, Comparative genomics

\footnotetext{
*Correspondence: alyssaburkhardt@gmail.com; kchilds@msu.edu; Frank.Martin@ars.usda.gov

${ }^{1}$ Crop Improvement and Protection Research Unit, USDA-ARS, Salinas, California, USA

${ }^{2}$ Department of Plant Biology and Center for Genomics-Enabled Plant

Science, Michigan State University, East Lansing, MI, USA
}

(c) The Author(s). 2019 Open Access This article is distributed under the terms of the Creative Commons Attribution 4.0 International License (http://creativecommons.org/licenses/by/4.0/), which permits unrestricted use, distribution, and reproduction in any medium, provided you give appropriate credit to the original author(s) and the source, provide a link to the Creative Commons license, and indicate if changes were made. The Creative Commons Public Domain Dedication waiver (http://creativecommons.org/publicdomain/zero/1.0/) applies to the data made available in this article, unless otherwise stated. 


\section{Background}

Macrophomina phaseolina is a haploid, clonally reproducing ascomycete fungus that causes damping off, stem rot, and charcoal rot on a wide range of over 500 host species including soybean, corn, wheat, and strawberry [1-3]. This pathogen is soilborne and can survive multiple growing seasons by forming resting structures called microsclerotia, which are melaninized structures formed from 50 to 200 cells [3]. Some studies have shown that the survival and symptom severity caused by $M$. phaseolina increases with warmer soil temperatures, ranging from 28 to $35^{\circ} \mathrm{C}[4,5]$. Typically, M. phaseolina has been thought to be a broad host range pathogen, with the same isolate being able to infect multiple plant species and thus posing a risk to growers planting a variety of crops in a field with a history of $M$. phaseolina $[1,6]$. However, recent studies suggest that $M$. phaseolina may exhibit some degree of host preference and that each isolate may not pose an equal risk to all crops [7]. Specifically, our recent work studying M. phaseolina collected from strawberry and other hosts around California support the hypothesis that some isolates of M. phaseolina exhibit a strong host preference toward strawberry [8].

Recently, an increased incidence of $M$. phaseolina in strawberry fields has been observed as growers shift away from using methyl bromide as a preplant fumigant as required by changes in pesticide regulations [8-10]. As a result, the 2.3-billon dollar, 15,000-ha strawberry industry in California [11] is under increasing threat from this pathogen that causes crown rot and death of the plant. Interestingly, this pathogen is found in cooler, coastal strawberry growing regions of California despite its documented preferences for warmer climates $[4,5]$. A large population study to understand potential regional and host differences of M. phaseolina from strawberry and other hosts growing mainly in California was conducted using SSR markers [12]. The results from an initial study using 65 SSR markers and 15 California isolates show that the majority of isolates recovered from strawberry cluster within a distinct clade [8]. A larger, unpublished study using 24 SSR markers and over 460 M. phaseolina isolates from California and other parts of the world support the grouping of most strawberry isolates into a single genotype (Marina Ramon and Frank Martin, personal communication). Understanding the genetics behind this single genotype grouping might lead to a better understanding of why some isolates of $M$. phaseolina exhibit a strong host preference for strawberry despite $M$. phaseolina being typically understood as a broad host range pathogen.

To begin to answer genomic questions, a high-quality, complete reference genome is needed to which other isolates can be compared. Prior to this study, the only published draft genome of M. phaseolina was sequenced from an isolate recovered from jute [1]. This isolate was sequenced using older technology, including Illumina and 454 , resulting in a $48.9 \mathrm{Mb}$ assembly consisting of 1506 contigs with an N50 of $151 \mathrm{~kb}$ [1]. The goal of the current study was not only to sequence isolates of $M$. phaseolina representing the main strawberry genotype and other genotypes, but also to use modern advances in sequencing technology, including long read PacBio sequencing, to provide a higher quality assembly with fewer fragments and more complete contigs. In recent years, the use of long read PacBio sequencing technology has lived up to the promise of delivering more complete genomes and has successfully been used to de novo assemble or dramatically improve the assembly of several plant-pathogenic fungi including Verticillium dahliae [13], Botrytis cinerea [14], Fusarium oxysporum [15], Magnaporthe oryzae [16], Sclerotinia sclerotiorum [17], and Colletotrichum higginsianum [18]. In fact, Faino et al. 2015 found that for $V$. dahliae an assembly based on PacBio data alone was of better quality than an assembly that was created from a hybrid of PacBio and Illumina data.

To achieve the goal of producing a very high-quality reference genome for M. phaseolina, an isolate recovered from strawberry in Santa Barbara county in 2011, 11-12, was selected to provide the DNA for the reference PacBio assembly because it was representative of the strawberry genotype and exhibited a host preference for strawberry [8]. In addition, an updated structural annotation of the genome was completed in this study using ten separate RNA-Seq libraries of the 11-12 fungal tissue grown in different conditions to generate strong transcript evidence.

Further improvement of the PacBio-based genome assembly was done using Illumina-based mate pair and paired-end sequencing in order to correct any sequencing errors and further improve the quality of the final assembly [19]. In addition, Dovetail Genomics (Santa Cruz, CA) Hi-C technology was used to join contigs into scaffolds, break any contigs that were erroneously joined in the initial assembly, and fill in gaps, thereby improving the long-range scaffolds of the assembly $[20,21]$. Because assemblies made with these new technologies provide significant advantages when investigating genome structure but may have sequence and structural bias when compared to Illumina-based assemblies, the genome of a second isolate of a different genotype named $\mathrm{Al}-1$, which was recovered from alfalfa in California in 2013, was also assembled as described above for the 11-12 isolate. Together, the data from these two isolates were assembled into high-quality genomes which were used to investigate how structural changes in the genome may provide the basis for potential mechanisms of genome evolution in this asexual fungus that could lead to host preference. 
Overall, the goals of the work in this manuscript were to 1) provide a complete high-quality genome assembly for Macrophomina phaseolina, 2) provide an updated annotation for M. phaseolina and 3) investigate the genomic changes that may have contributed to strawberry host preference of some isolates of $M$. phaseolina.

\section{Results}

\section{Genome assembly}

In the process of generating the final assemblies for isolates $11-12$ and $\mathrm{Al}-1$, several methods and iterations of assembly were used to generate the highest quality assembly. Before the FALCON-based assemblies were selected as the final assembly, the HGAP assembly pipeline was used to generate an assembly for the 11-12 isolate with the PacBio data, but it had a lower N50 (3.3 $\mathrm{Mb})$ than the FALCON assembly $(\mathrm{N} 50=4.3 \mathrm{Mb})$ and did not run successfully with input data for isolate Al-1. The Genome Finishing Module of the CLC Genomics Workbench (Qiagen, Redwood City, CA) was also used to assemble both genomes using the PacBio data, but in both cases resulted in an assembly with a shorter total length (44 Mb) and over 120 contigs in each assembly. After the FALCON-based assemblies were selected as the best PacBio-based assemblies, a polishing run with PILON using paired-end and mate-pair Illumina reads greatly improved the base calling, with 113,895 and 73 , 557 SNP and indel changes made after the first PILON run for the $\mathrm{Al}-1$ and the 11-12 assemblies, respectively. A second PILON polishing step was done and yielded a higher percentage of Illumina reads mapping. In this step, 3984 and 3784 additional SNP changes were made by PILON in the Al-1 and the 11-12 assemblies, respectively, resulting in the final highest quality, polished FALCON-based assembly.

The final PILON assemblies were further improved using Dovetail HiRise with PacBio gapfilling to combine some contigs into scaffolds. For 11-12, the input assembly of 102 contigs was broken once and joined 19 times, resulting in 84 total contigs/scaffolds. The joins made by the initial Dovetail HiRise scaffolding were made by adding $100 \mathrm{Ns}$ at the junction, but 6 of these joins were subsequently filled in with the PacBio data from the initial sequencing, leaving 13 total gaps filled with 25$100 \mathrm{Ns}$. For Al-1, the input assembly of 27 contigs was not broken, but a single join was made with Dovetail HiRise resulting in 26 final contigs. The gap created by this contig joining was eliminated by the PacBio gapfill resulting in a final assembly of isolate $\mathrm{Al}-1$ with no gaps and no Ns. To investigate the identity of the smaller $(<$ $100 \mathrm{~kb}$ ) contigs in each assembly, the assemblies were aligned to themselves using a BLAST-based tool in CLC Genomics Workbench, and contigs that were $99.5 \%$ contained within another contig and had greater than 99.5\% identity to that contig were considered assembly errors and were eliminated along with contigs less than 1000 bp. After the Dovetail and CLC analyses of the genome, the final contig/scaffold counts were 60 and 18 for the 11-12 and Al-1 genomes, respectively. All of the contig/ scaffolds and their lengths can be found in Additional file 1: Table S1. For ease of reference, the large segments of DNA in the final assemblies, which are largely gap-free, have all been named as "Contig \#" with the Contig 1 being the longest contig for each assembly and Contig $n$ being the shortest contig for each assembly with $n$ representing the total number of contigs. The genome statistics for the final assemblies can be found in Table 1.

High-quality, nearly complete assemblies were generated for both the M. phaseolina isolate from strawberry, 11-12, and the isolate from alfalfa, Al-1 (Table 1). Both isolates had very high N50 scores, at $4.3 \mathrm{Mb}$ and $5.0 \mathrm{Mb}$ for the 11-12 and Al-1 isolate, respectively. The sequence coverage of the $M$. phaseolina genomes from strawberry and alfalfa was $150 x$ for the PacBio reads and over 200x for the Illumina reads. The distribution of the lengths of the main contigs for 11-12 and Al-1 indicate that there are 13 large contigs that are all $870 \mathrm{~kb}-6.8$ $\mathrm{Mb}$. The smaller 47 contigs of 11-12 range from 4726 to 99,276 bp with Contig 15 ( $94,974 \mathrm{bp}$ ) being the mitochondrial genome. The 5 smaller contigs of $\mathrm{Al}-1$ range from 2215 to $49,130 \mathrm{bp}$ with Contigs 14, 16, 17, and 18 representing the mitochondrial genome. No telomeres were detected in the 11-12 assembly, but 7 out of 14 nuclear contigs of the Al-1 assembly had a telomere on one end. When mapping the paired-end Illumina reads generated from the 11-12 isolate and the Al-1 isolate

Table 1 Genome statistics for Macrophomina phaseolina isolates from strawberry that represent the strawberry genotype (11-12) and isolates recovered from diseased alfalfa (Al-1) and jute that represent other genotypes

\begin{tabular}{|c|c|c|c|c|c|c|c|c|c|c|}
\hline Isolate & $\begin{array}{l}\text { Genome Size } \\
\text { (Mb) }\end{array}$ & $\begin{array}{l}\text { \# of Contigs/ } \\
\text { Scaffolds }\end{array}$ & $\begin{array}{l}\mathrm{N} 50 \\
\mathrm{Mb}\end{array}$ & L75 & $\begin{array}{l}\text { Long Contig } \\
\mathrm{Mb}\end{array}$ & $\begin{array}{l}\% 11-12 \text { reads } \\
\text { mapped }\end{array}$ & $\begin{array}{l}\% \text { Al-1 reads } \\
\text { mapped }\end{array}$ & $\begin{array}{l}\text { BUSCO } \\
\text { euk }\end{array}$ & $\begin{array}{l}\text { BUSCO } \\
\text { fungi }\end{array}$ & $\begin{array}{l}\text { \# of } \\
\text { genes }\end{array}$ \\
\hline $11-12$ & 51.3 & $60^{\mathrm{a}}$ & 4.3 & 8 & 6.8 & $96.4 \%$ & $93.7 \%$ & $98.3 \%$ & $99.3 \%$ & 14,103 \\
\hline $\mathrm{Al}-1$ & 49.8 & $18^{\mathrm{b}}$ & 5.0 & 8 & 6.8 & $85.0 \%$ & $98.1 \%$ & $98.3 \%$ & $99.0 \%$ & 13,443 \\
\hline Jute $e^{c}$ & 48.9 & 1506 & 0.15 & 205 & 1.1 & $84.9 \%$ & $93.5 \%$ & $98.3 \%$ & $98.3 \%$ & 14,249 \\
\hline
\end{tabular}

${ }^{\mathrm{a}}$ One contig represents the mitochondrial genome

${ }^{b}$ Four contigs represent the mitochondrial genome

Islam et al. [1] 
back to the PacBio-based assemblies of their respective isolates, $>96 \%$ of the trimmed reads mapped back to the assembly, indicating that the assemblies were of high quality and that the base calling could be largely confirmed with a different sequencing technology. A lower percentage of the paired-end reads mapped from the isolate of origin to the other sequenced isolate, indicating some genomic divergence between the isolates. Overall, the assemblies had good BUSCO scores using both the eukaryotic $(<98 \%)$ and the fungal $(<99 \%)$ databases, indicating that the core set of genes were fully present and correctly assembled within each assembly (Table 1).

Transposable elements (TEs) were identified in each genome using RepeatMasker (Table 2). Overall, the total number of transposable elements identified in each genome assembly was similar, with 2882 TEs identified in the 11-12 assembly and 2703 TEs in the Al-1 assembly. Major types of TEs identified included Class I retrotransposons, including LTRs (long terminal repeats) and LINES (long interspersed nuclear elements) as well as Class II transposons including DNA transposons and helitrons [22]. Some groups of transposons were unique to the 11-12 isolate, including the L2 category of LINE transposons that were found on 8 of the 13 main contigs as well as the CRE type of LINE transposon that was only found on Contig 8. The Penelope type of LINE was only present in the 11-12 genome and is a unique type of TE that is typically associated with animal genomes [23]. Within the 11-12 genome, this TE type was more prevalent in the smaller contigs than in the 13 main contigs. Both genomes had a large number of Copia and Gypsy LTRs, which are known to be both abundant and diverse in fungal genomes [24]. In contrast, only the 11-

Table 2 Transposable elements identified in Macrophomina phaseolina genomes from an isolate recovered from strawberry that represents the strawberry genotype (11-12) and an isolate recovered from alfalfa (Al-1) that represents another genotype

\begin{tabular}{lcc}
\hline Transposable Element Category & $11-12$ & Al-1 \\
\hline DNA & 490 & 398 \\
LINE/Penelope & 109 & \\
LINE/Tad1 & 269 & 179 \\
LINE/CRE & 1 & \\
LINE/L2 & 12 & 158 \\
LINE (other) & 189 & 385 \\
LTR/Copia & 550 & 1522 \\
LTR/Gypsy & 1173 & \\
LTR/ERV4 & 17 & 43 \\
LTR (other) & 59 & 15 \\
RC/Helitron & 13 & \\
\hline
\end{tabular}

LINE Long interspersed nuclear element $L T R$ Long terminal repeat $R C$ Rolling circle
12 genome contained endogenous retrovirus 4 (ERV4) LTRs, which were only found within the smaller contigs of the genome and have previously been identified in other fungal genomes [25].

Genome annotation for M. phaseolina isolates 11-12 and Al-1 MAKER was used to annotate the genomes of 11-12 and Al-1 using a set of protein evidence from closely related fungi and an RNA-Seq based transcript dataset from the 11-12 isolate. MAKER used several de novo gene predictors including AUGUSTUS, SNAP, and GENEMARK to begin the annotation process for these two M. phaseolina isolates. A final list of MAKER standard genes was created from the predicted genes that had Pfam support and or transcript evidence. In total, the 11-12 isolate contained 14, 103 annotated genes and the $\mathrm{Al}-1$ isolate contained 13,443. For the $\mathrm{Al}-1$ genome, all of these annotated genes are on the 13 main contigs, with no MAKER-annotated genes found on the $41.5 \mathrm{~kb}$ contig or the four contigs representing the mitochondrial genome. Alternatively, there are 146 annotated genes on the 47 smaller contigs ( 4726 to $99,276 \mathrm{bp}$ ) of 11-12, ranging from 0 to 18 annotated genes per contig. The functional (Pfam) annotations of these genes identified on the 11-12 small contigs included helicases, retrotransposons, translation initiation factors, oxidoreductases, and aspartyl proteases.

The carbohydrate-active enzyme composition of a fungus can be used to determine its ecological niche and host specificity. To compare the carbohydrate-active enzymes from the two sequenced M. phaseolina isolates with other plant pathogenic and non-plant associated fungi, the carbohydrate-active enzymes encoded by each fungus in the comparison were classified using the CAZy database (Table 3). Carbohydrate-active enzymes are classified into six families, and the observed enzyme compositions are conserved within the M. phaseolina isolates. As expected, this comparison shows that the polysaccharide lyase (PL) gene family expanded in the plant pathogenic fungi relative to the non-plant associated fungi (e.g., A. niger and $N$. crassa). The PLs encoded in the $M$. phaseolina cleave different forms of pectins, such as pectate lyases in the PL1, PL3, and PL9 families, and rhamnogalacturonan lyases in the PL4 family. Additionally, the glycoside hydrolase (GH) enzymes (e.g., GH88 and GH105) that degrade the products generated by PL enzymes are also found in the M. phaseolina genomes. The enhanced capacity in the pectinolytic machinery suggests strong plant cell wall degradation activity that likely enables their necrotrophic infection and colonization of plant tissue.

Genome sequence comparison between 11-12 and Al-1 reveals large-scale genome rearrangement

When comparing the full genome assembly of 11-12 to $\mathrm{Al}-1$, large scale structural rearrangements and smaller 
Table 3 Carbohydrate-Active Enzymes of Macrophomina phaseolina isolates, 11-12, Al-1, and jute, and other fungi including Aspergillus niger, Neurospora crassa, Fusarium vertcilliodes, and Verticillium dahliae

\begin{tabular}{|c|c|c|c|c|c|c|c|}
\hline \multirow{4}{*}{$\begin{array}{l}\text { CAZY } \\
\text { families }\end{array}$} & \multicolumn{7}{|l|}{ Fungal Species } \\
\hline & M. phaseolina & M. phaseolina & M. phaseolina & A. niger & N. crassa & F. verticillioides & V. dahliae \\
\hline & \multicolumn{7}{|c|}{ Fungal Isolate Names } \\
\hline & $11-12$ & $\mathrm{Al}-1$ & MPI-SDFR & ATCC 1015 & OR74A & 7600 v2 & VdLs.17 \\
\hline$\overline{A A^{a}}$ & 170 & 161 & 172 & 113 & 75 & 145 & 104 \\
\hline $\mathrm{CBM}^{\mathrm{b}}$ & 23 & 24 & 26 & 29 & 22 & 43 & 31 \\
\hline $\mathrm{CE}^{\mathrm{C}}$ & 120 & 117 & 124 & 91 & 46 & 140 & 77 \\
\hline $\mathrm{GH}^{\mathrm{e}}$ & 327 & 335 & 336 & 310 & 222 & 414 & 292 \\
\hline GH88 & 1 & 1 & 1 & 1 & 0 & 2 & 3 \\
\hline GH115 & 2 & 2 & 2 & 0 & 1 & 2 & 4 \\
\hline$G T^{d}$ & 128 & 127 & 126 & 148 & 116 & 190 & 120 \\
\hline$P L^{f}$ & 26 & 26 & 29 & 11 & 4 & 24 & 35 \\
\hline PL1 & 9 & 8 & 9 & 8 & 1 & 11 & 16 \\
\hline PL3 & 10 & 11 & 11 & 0 & 1 & 7 & 11 \\
\hline PL4 & 5 & 5 & 5 & 2 & 1 & 3 & 4 \\
\hline PL9 & 1 & 1 & 1 & 0 & 0 & 2 & 2 \\
\hline
\end{tabular}

${ }^{\mathrm{a}} A A$ Auxiliary activities

${ }^{\mathrm{b}} C B M$ carbohydrate-binding modules

${ }^{C} C E$ Carbohydrate esterase

${ }^{\mathrm{d}} G T$ glycosyltransferases

${ }^{\mathrm{e}} \mathrm{GH}$ glycoside hydrolases

${ }^{\mathrm{f}} P L$ polysaccharide lyases

scale indels and SNPs were observed. The dot plot from a MUMmer analysis visualized using Assemblytics indicates that the genomes are largely collinear (Fig. 1). Some contigs have been inverted or translocated. For example, a portion of Contig 2 of the 11-12 assembly is collinear and found inverted and translocated to Contig
8 of the Al-1 assembly. Large portions of 11-12 Contigs 3,6 , and 7 are also shown to be collinear and translocated to a different contig of the Al-1 isolate. Of the larger contigs, Contig 8 shows the most changes with large portions broken and or translocated and inverted in the $\mathrm{Al}-1$ assembly relative to the 11-12 assembly.

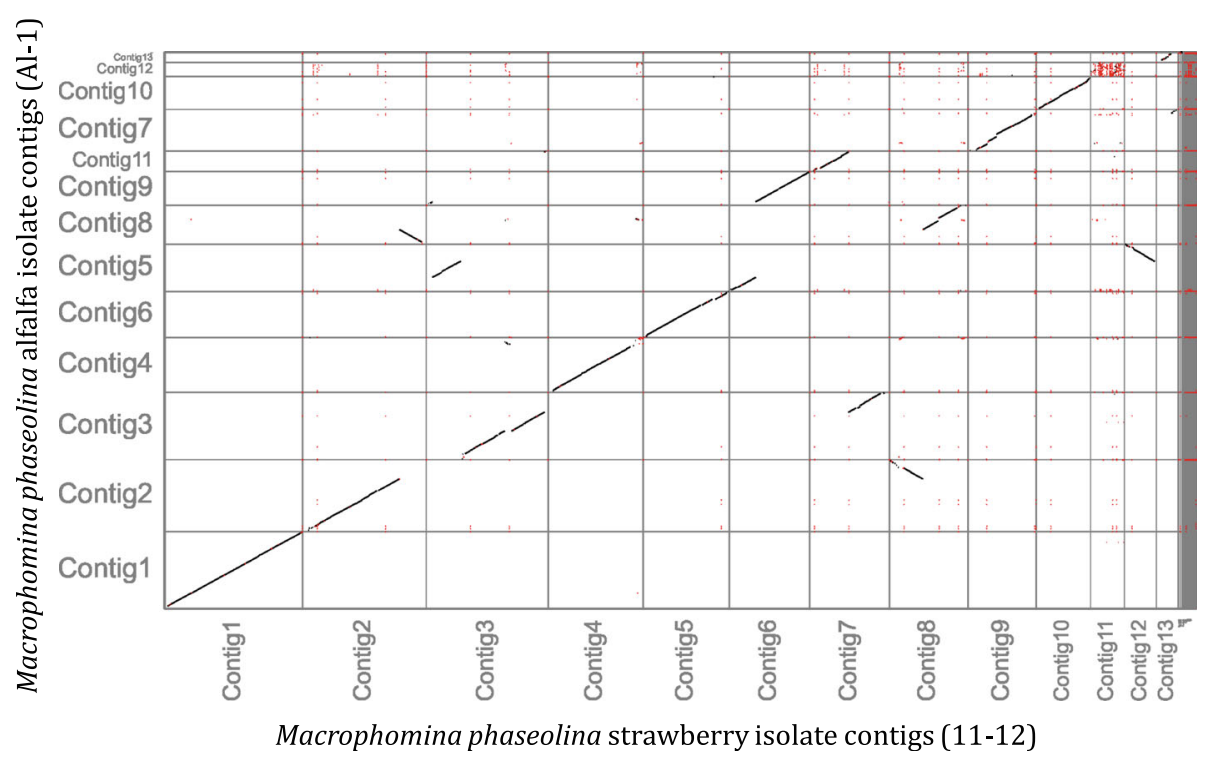

Fig. 1 MUMmer-based Assemblytics dot plot comparing the genomes of Macrophomina phaseolina isolates 11-12 (strawberry genotype) and Al1 (non-strawberry genotype) 
Similar pairwise whole-genome comparisons were made between the 11-12 assembly and the Illumina assemblies of other $M$. phaseolina isolates using MUMmer and Assemblytics. Overall, the genomes of all isolates, either pathogenic on strawberry or nonpathogenic on this host, were collinear with the 11-12 strawberry isolate, with some small segments of the genome showing translocations or inversions but with none of these rearrangements being consistently associated with the strawberry genotype. In general, the genomes of isolates that were nonpathogenic on strawberry exhibited more insertions and deletions compared to the 11-12 assembly, with a higher proportion of deletions, compared to those identified among the isolates pathogenic on strawberry. However, a couple of pathogenic isolates also had a high number of insertions and deletions compared to the 11-12 assembly and some nonpathogenic isolates had very few indels, indicating that using the trends of indels alone is not an accurate predictor of pathogenicity on strawberry. The different sequencing technologies used for the genome assemblies may affect the analyses given that the 11-12 long-read assembly was composed largely of 13 contigs while the Illumina-based assemblies of the other M. phaseolina isolates contain over 1000 contigs each.

A progressive MAUVE analysis between the 11-12 and $\mathrm{Al}-1$ assemblies revealed translocations and inversions as well as several syntenic blocks between the two genomes (Fig. 2). Contig 1 of both assemblies, which is over $6 \mathrm{Mb}$ and the longest contig of each assembly, has two syntenic blocks. Some portions of Contig 8 of the Al-1 genome are similar to Contig 2 of the 11-12 assembly but are inverted. The third contig of each assembly has partial synteny, but a portion of the 11-12 contig can be found predominantly on Al-1 Contig 5 (inverted) and to a lesser extent Contigs 4 and 9 (Fig. 1). A portion of the Al-1 genome syntenic to Contig 6 of the 11-12 assembly is inverted and present on Contig 5 of the Al-1 assembly along with portions of 11-12 Contigs 3 and 12. Several blocks of the 11-12 Contig 7 can be found on the Al-1 Contig 3 and 11. Contig 8 of 1112 has a region from position 359,168 to 449,477 that appears to be a unique sequence region without a clear corresponding match in the Al-1 assembly. Contig 11 of the 11-12 assembly also seems to contain unique genomic regions and does not have many syntenic regions with Al-1.

\section{Identification of gene clusters from M. phaseolina unique to the main strawberry genotype}

An analysis of all the orthologous genes, including the jute $M$. phaseolina assembly [1] as well as seventeen other taxa, including several other fungi within the Dothideomycetes class (Additional file 2: Table S2), were analyzed with OrthoFinder. In the resulting phylogenetic tree, the isolates of $M$. phaseolina were tightly grouped with each other and with the other members of the Botryosphaeriales order, as expected (Fig. 3; Percentage of shared orthologous genes between species is in Additional file 3: Table S3). This phylogeny also indicated isolates of $M$. phaseolina from alfalfa and jute were more closely related to each other than to the strawberry isolate, providing additional support that the host specificity aspect of the strawberry isolate genotype may be associated with evolutionary divergence. This phylogeny helped to inform the selection of isolates that were used to create OrthoVenn2 comparisons with smaller groupings of isolates. The OrthoFinder analysis also identified 2 unique orthogroups for the main strawberry genotype of $M$. phaseolina with 6 genes represented. The isolate from alfalfa did not have any specific orthogroups and the isolate from jute had 4 specific orthogroups.

In an analysis with OrthoVenn2 using the M. phaseolina protein sequences from strawberry, jute, and alfalfa isolates, 80 clusters containing a total of 223 proteins were found to be unique to the strawberry genotype with 1338 singletons also identified as being unique (Fig. 4a). Within the group of 80 unique clusters, enriched GO slim terms, included those associated with a molecular function (nucleotide binding, oxidoreductase activity, hydrolase activity, and

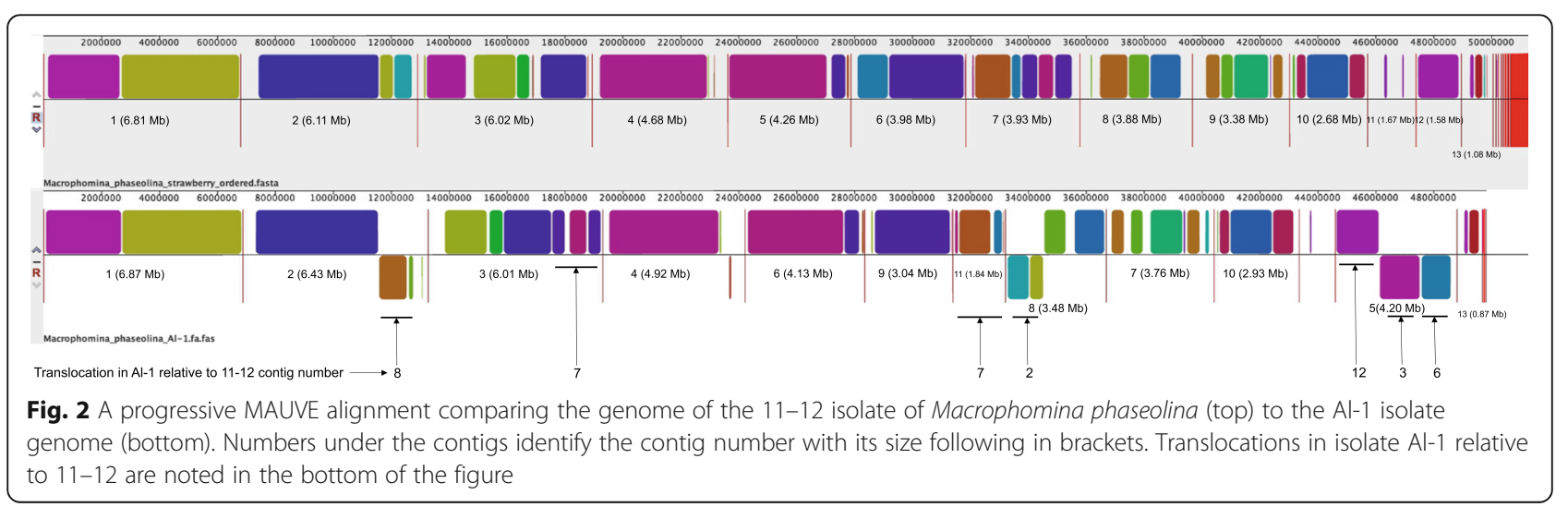




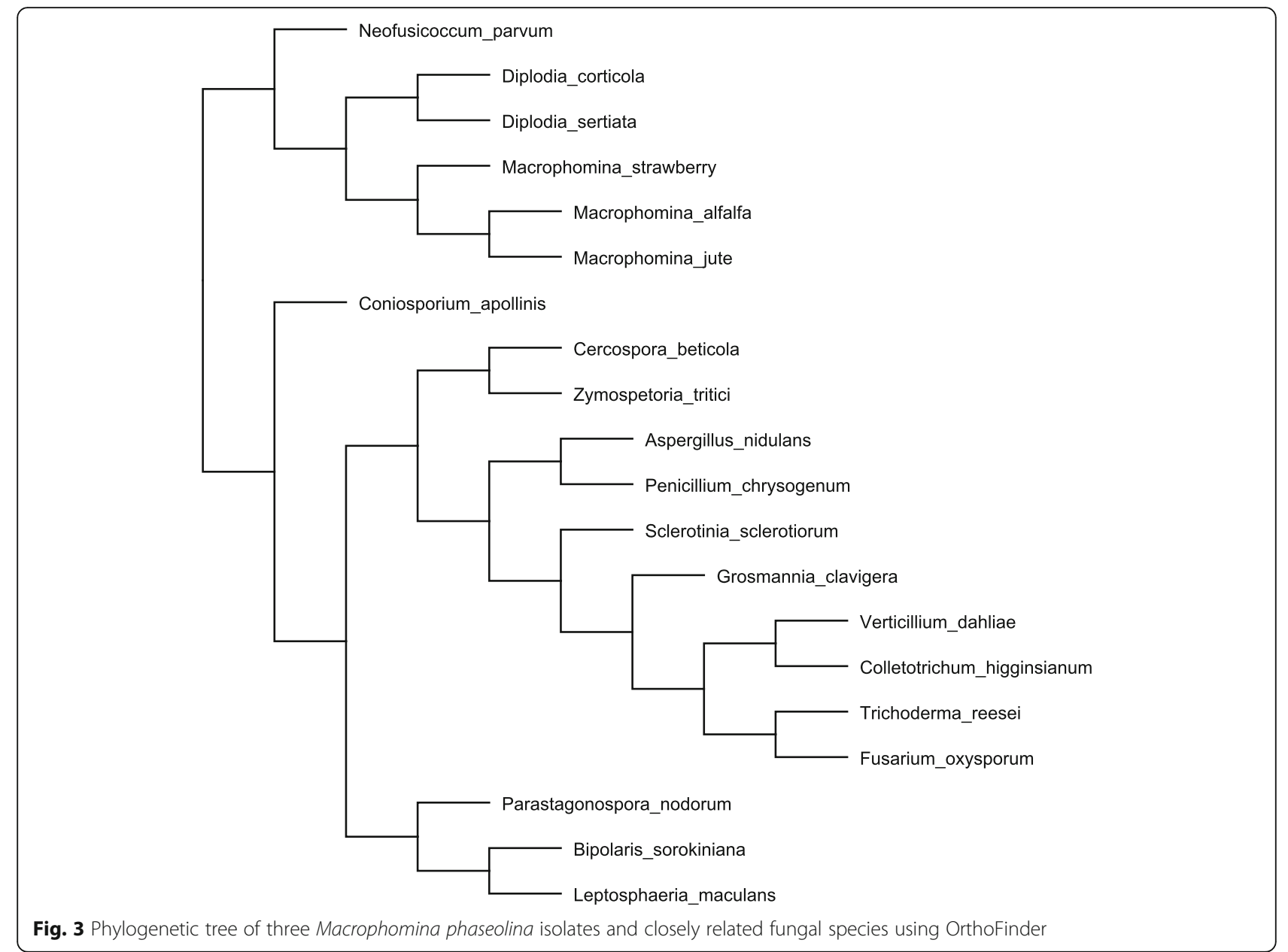

cofactor binding) and those associated with biological process (pyrimidine nucleotide biosynthetic process, cellular aromatic compound metabolic process, and others). Common among all three M. phaseolina isolates were 10, 678 orthologous clusters with enriched GO terms associated with oxidoreductase activity (115 groups, $p$-value $2 \times$ $10^{-6}$ ) and terpenoid biosynthetic process (48 groups, $p$ value $6.5 \times 10^{-5}$ ). These two enriched GO terms provide support and additional insight into the potential infection mechanisms of M. phaseolina relative to other fungi. Oxidoreductase activity in terms of hydrogen peroxide production has been positively correlated with virulence in some strains of M. phaseolina on chickpea and sunflower [26] and terpene-derived secondary metabolites produced by filamentous fungi like Fusarium have been implicated in toxicity to plants [27].

An analysis between the selected fungi within the Botryosphaeriales order with OrthoVenn2 showed that the $M$. phaseolina isolate from strawberry had 75 unique clusters of orthologous genes with enriched GO slim terms including phosphorus metabolic process and oxidoreductase activity (Fig. 4b). The strawberry isolate also had 1231 singletons without an orthologous gene. Within the same OrthoVenn2 analysis, the three $M$. phaseolina isolates uniquely shared 1729 orthologous groups which had enriched GO terms including the molecular function of oxidoreductase activity ( 80 groups, $p$ value $<1 \times 10^{-9}$ ) and the biological processes of pathogenesis (29 groups, $p$-value $\left.5 \times 10^{-8}\right)$ and terpenoid biosynthetic process (14 groups, $p$-value $3.6 \times 10^{-4}$ ). All of the fungi in this analysis shared 5739 orthologous clusters, which included enriched GO terms including the biological processes of pathogenesis (48 groups, $p$-value $\left.9 \times 10^{-10}\right)$, terpenoid biosynthetic process (19 groups, $p$ value $\left.2.4 \times 10^{-9}\right)$, and vesicle-mediated transport (44 groups, $p$-value $2.8 \times 10^{-7}$ ). Several $\mathrm{GO}$ terms related to oxidoreductase activities were also enriched among all of the fungi in this analysis.

Another OrthoVenn2 analysis among genera of fungi that commonly infect strawberry - Macrophomina, Fusarium, Colletotrichum, Verticilium, and Sclerotinia - had 410 unique orthologous groups identified in the M. phaseolina isolate from strawberry along with 4110 singletons (Fig. 4c). Enriched GO terms for the unique orthologous groups 
a)

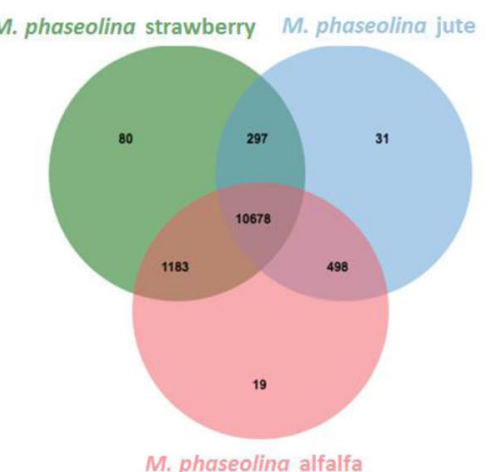

b)

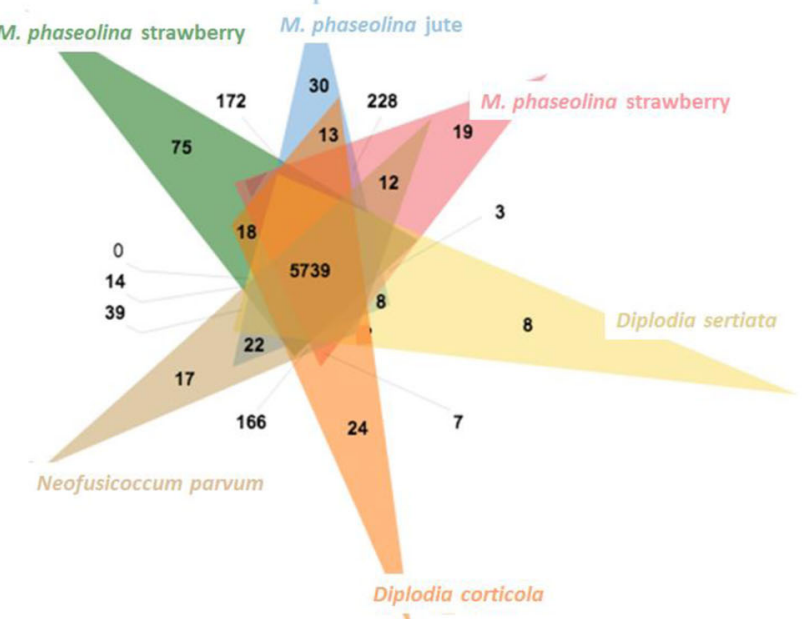

c)

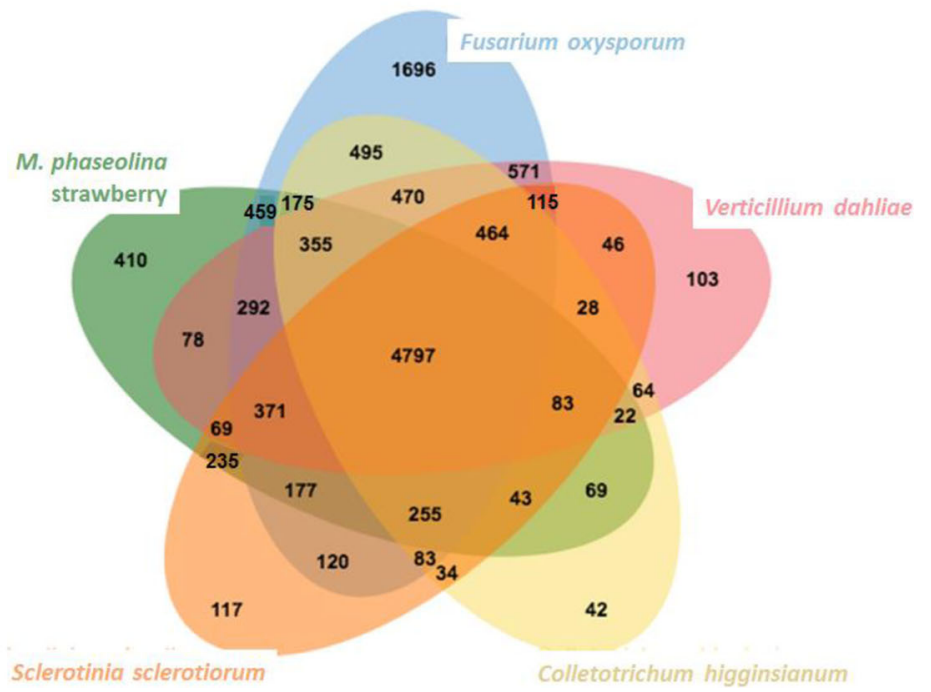

Fig. 4 OrthoVenn diagram comparisons of Macrophomina phaseolina isolate 11-12 to a) two other M. phaseolina isolates from jute and alfalfa (Al-1). $\mathbf{b}$ other Botryosphaeriales fungi and $\mathbf{c}$ ) other fungi that commonly infect strawberry

include the molecular function of oxidoreductase activity (15 groups, $p$-value $1.3 \times 10^{-12}$ ) and the biological processes of shamixanthone biosynthetic process (3 groups, $p$-value $\left.2.5 \times 10^{-4}\right)$ and terpenoid biosynthetic process (5 groups, $p$-value $\left.2.5 \times 10^{-4}\right)$. All five fungi shared enriched GO terms including those associated with the biological process of transmembrane transport $(60$ groups, $p$-value $\left.1.5 \times 10^{-14}\right)$, the molecular function of 
zinc ion binding (18 groups, p-value $1.1 \times 10^{-13}$ ), and the biological process of pathogenesis (31 groups, $p$-value $8.4 \times 10^{-10}$ ). Also enriched were genes with GO terms that are associated with the breakdown of cell walls and thus perhaps pathogenicity, including polysaccharide catabolic process (14 groups, $p$-value $8.3 \times 10^{-8}$ ), cellulose catabolic process (11 groups, $p$-value $1.5 \times 10^{-6}$ ), and $x y-$ lan catabolic process (7 groups, $p$-value $1 \times 10^{-5}$ ).

Identification of candidate $M$. phaseolina genes associated with isolates pathogenic on strawberry

A comparative genomics approach using the annotated genome from the strawberry genotype was used to identify candidate genes that may be unique to $M$. phaseolina isolates that are capable of infecting strawberry. The sequencing reads from each of the 30 other sequenced isolates of M. phaseolina - including isolates that were both pathogenic and not pathogenic on strawberry were perfectly mapped to the 11-12 genome (Table 4). These 30 isolates were selected because they represented the breadth of $M$. phaseolina genetic diversity and were used in a companion project to find a genomic locus that was used as a diagnostic tool to detect isolates pathogenic on strawberry [28]. In order to focus on differences only within the annotated genes in this study, HT-Seq was used to count the number of reads that mapped to each annotated gene model. Genes with no or low counts in the isolates that were not pathogenic on strawberry and with high counts in the isolates that were considered pathogenic on strawberry were selected

Table 4 All isolates of Macrophomina phaseolina in addition to isolates 11-12 and Al-1 that were sequenced with Illumina and pathogenicity tested

\begin{tabular}{|c|c|c|c|c|}
\hline Isolate & Host of isolate origin & Location of isolate origin (California) & Pathogenic on Strawberry & NCBI Data Accession \\
\hline $11-21$ & Cantaloupe & Los Banos & No & SAMN09764508 \\
\hline $11-22$ & Cantaloupe & Los Banos & No & SAMN09764509 \\
\hline $13-10$ & Watermelon & Imperial County & No & SAMN09764510 \\
\hline $13-11$ & Watermelon & Imperial County & No & SAMN09764511 \\
\hline 14-177 & Sunflower & Imperial County & No & SAMN09764512 \\
\hline $14-24$ & Strawberry & Stanislaus County & No & SAMN09764513 \\
\hline $14-26$ & Strawberry & Ventura County & No & SAMN09764514 \\
\hline $14-27$ & Strawberry & Ventura County & No & SAMN09764515 \\
\hline $14-4$ & Almond & Fresno & No & SAMN09764516 \\
\hline $14-45$ & Lima bean & Santa Clara County & No & SAMN09764517 \\
\hline $14-48$ & Lima bean & Santa Clara County & No & SAMN09764518 \\
\hline $16-13$ & Strawberry & Santa Barbara County & No & SAMN09764519 \\
\hline $11-14$ & Strawberry & Ventura County & Yes & SAMN09764520 \\
\hline $11-5$ & Strawberry & California nursery & Yes & SAMN09764521 \\
\hline $12-27$ & Strawberry & Santa Cruz County & Yes & SAMN09764522 \\
\hline $13-30$ & Strawberry & Monterey County & Yes & SAMN09764523 \\
\hline $13-45$ & Strawberry & Monterey County & Yes & SAMN09764524 \\
\hline 14-134 & Strawberry & Santa Clara County & Yes & SAMN09764525 \\
\hline $14-140$ & Pepper & Santa Clara County & Yes & SAMN09764526 \\
\hline 14-170 & Strawberry & Oxnard County & Yes & SAMN09764527 \\
\hline $14-20$ & Strawberry & Ventura County & Yes & SAMN09764528 \\
\hline $14-21$ & Strawberry & Ventura County & Yes & SAMN09764529 \\
\hline $14-22$ & Strawberry & Stanislaus County & Yes & SAMN09764530 \\
\hline $14-62$ & Strawberry & Santa Maria County & Yes & SAMN09764531 \\
\hline $15-47$ & Strawberry & Salinas & Yes & SAMN09764532 \\
\hline $15-69$ & Strawberry & Salinas & Yes & SAMN09764533 \\
\hline $15-87$ & Strawberry & California nursery & Yes & SAMN09764534 \\
\hline $15-89$ & Strawberry & California nursery & Yes & SAMN09764535 \\
\hline $16-14$ & Strawberry & San Luis Obispo & Yes & SAMN09764536 \\
\hline $16-15$ & Strawberry & San Luis Obispo & Yes & SAMN09764537 \\
\hline
\end{tabular}


after the first round of read mapping. Within CLC Workbench, BLAST was used to compare the gene model from the 11-12 isolate to similar genes in the other isolates to identify potential truncations or SNPs that could eliminate a candidate if these mutations were to cause significant changes in the predicted protein sequence among isolates in the main strawberry genotype. Non-synonymous mutations in candidate genes among the isolates non-pathogenic on strawberry allowed some candidate genes that had a high mapped read count to a candidate gene model in 11-12 to remain as pathogenicityassociated gene candidates. After this selection process, 10 candidate genes (Table 5) were identified, and all of them are located within a $\sim 200 \mathrm{~kb}$ region of Contig 8 of the 11-12 assembly. Interestingly, this region is broken in a dotplot alignment between 11-12 and Al-1 (Fig. 1) and shows rearrangements and indels within the MAUVE alignment (Fig. 2).

The candidate genes identified in Table 5 were typically absent in all 13 of the nonpathogenic isolates and present in all 19 of the isolates of the strawberry genotype pathogenic on this host except in the case of isolates 14-26 and 14-27. These isolates were recovered from diseased strawberry plants in Ventura, California, are not in the strawberry genotype, and were found to be nonpathogenic on strawberry in the toothpick inoculations, yet had sequences of candidate genes that were identical to those found in 11-12. Several of these genes, including M11_12_v1_01446, M11_12_v1_01448, M11_ 12_v1_01460, M11_12_v1_01490, M11_12_v1_01493, and M11_12_v1_01501 were also listed as a member of a unique orthologous grouping or as singletons for $M$. phaseolina of strawberry in all the OrthoVenn2 comparisons. Additionally, for some candidate genes - M11 12_v1_01458, M11_12_v1_01460, M11_12_v1_01463, and M11_12_v1_01466 - isolates nonpathogenic on strawberry, Al-1 and 14-4, had similar gene sequences, but SNPs or indels changed the predicted protein sequence when compared to that of 11-12. For gene M11_12_v1_01493, the predicted proteins from all the nonpathogenic isolates except for 14-26 and 14-27 were truncated compared to the 11-12 gene model and were predicted to be metalloproteinases while the full-length gene model from 11-12 was predicted to encode a membrane dipeptidase. This is also the only gene among the candidate genes that was predicted by SignalP to have a signal peptide. When looking at the transcript support for each candidate gene model, all the genes except for M11-12_v1_01448, M11_12_v1_01493, and M11-12_ v1_01501 had full or partial support from the transcripts predicted by Trinity using the 11-12 RNA-Seq datasets provided during the gene annotation. Furthermore, when examined individually, many of the RNA-Seq datasets provided support for the expression of the candidate genes, with M11_12_v1_01446, M11_12_v1_01448, M11_ 12_v1_01463, and M11_12_v1_01501 being highly expressed in all the transcript datasets. A couple of genes, M11_12_v1_01455 and M11_12_v1_01493, had higher gene expression in the dataset from the crown tissue medium compared to other growth conditions, but additional experiments are needed to confirm the significance of this observation.

\section{Discussion}

Overall, this study provides two high-quality assemblies for $M$. phaseolina and explores genetic differences between $M$. phaseolina isolates collected from different hosts. The use of third generation sequencing technology, including long read PacBio sequencing, allowed for a genome that was more complete and contiguous compared to the previously published genome that was assembled using older short-read technology [1]. Furthermore, the assemblies presented in this manuscript are of very high quality, with thorough genome polishing completed using PILON with Illumina-based mate pair and paired-end libraries. The improved sequencing

Table 5 Candidate genes associated with isolates in the main strawberry genotype that are aggressive on strawberry

\begin{tabular}{lccclll}
\hline Candidate Gene & Start & Stop & Gene Length & Pfam Domain & Signal Peptide \\
\hline M11_12_v1_01446 & 467,679 & 468,583 & 904 & Ribosomal protein L36 & no \\
M11_12_v1_01448 & 458,879 & 462,158 & 3279 & Myb-like DNA-binding domain & no \\
M11_12_v1_01455 & 468,323 & 470,605 & 2282 & NA & no \\
M11_12_v1_01458 & 523,857 & 525,833 & 1976 & $\begin{array}{l}\text { Major Facilitator Superfamily, Sugar (and other) } \\
\text { transporter, Organic Anion Transporter Polypeptide (OATP) family }\end{array}$ & no \\
M11_12_v1_01460 & 530,438 & 531,136 & 698 & Fungal Zn(2)-Cys(6) binuclear cluster domain, Zinc knuckle & no \\
M11_12_v1_01463 & 516,694 & 519,283 & 2589 & Aldehyde dehydrogenase family & no \\
M11_12_v1_01466 & 519,472 & 521,373 & 1901 & Cytochrome P450 & no \\
M11_12_v1_01490 & 654,497 & 656,140 & 1643 & NA & yes \\
M11_12_v1_01493 & 623,466 & 624,632 & 1166 & Membrane dipeptidase (Peptidase family M19) & no \\
M11_12_v1_01501 & 655,801 & 656,697 & 896 & Protein of unknown function (DUF3408) & \\
\hline
\end{tabular}


technology was used at a much higher coverage $(150 \mathrm{x}$ for PacBio and 200x for Illumina) compared to the $66 \mathrm{x}$ coverage for the jute assembly. Beyond using advancements in sequencing technology, this work also confirmed the accuracy of assemblies with new methods in genome scaffolding from Dovetail Genomics, which used $\mathrm{Hi}-\mathrm{C}$ proximity ligation, the HiRise software, and PacBio gapfilling. This resulted in a final assembly with no gaps within the contigs in the case of the $\mathrm{Al}-1$ genome and 13 gaps in the case of the 11-12 genome. The total genome size for all three isolates was similar, with the strawberry isolate at $51.3 \mathrm{Mb}$, the alfalfa isolate at 49.8 $\mathrm{Mb}$, and the jute isolate at $48.9 \mathrm{Mb}$. Compared to the 454 and Illumina short read technology used in the assembly of the jute isolate [1], the use of the long-read PacBio technology and Dovetail scaffolding of Hi-C data greatly decreased the total number of scaffolds, improved the L75, and increased the length of the longest contig, as shown in Table 1. Overall, this approach has produced very high-quality genomes with an N50 of 4.3 and $5.0 \mathrm{Mb}$ for the strawberry and alfalfa isolates, respectively. This is a significant improvement over the previous reference assembly of $M$. phaseolina of an isolate recovered from jute, which had an N50 of $151 \mathrm{~kb}$ [1].

While the assemblies presented in this work are very high quality and are an improvement over the published genome from a jute isolate, there are areas where future work could improve the quality of the strawberry genotype assembly to make a fully complete chromosomelevel assembly. First, one shortcoming of this study is that FALCON, an assembler designed for diploids, was used to assemble a haploid genome [29]. In this case, the primary contig file was used as the basis for the final assembly, but a smaller associate contig file was also produced by the assembler which contained $\sim 50$ small contigs per assembly that were each $<70 \mathrm{~kb}$. These contigs were compared by BLAST to the final assembly and were found to be fully contained within the primary contigs and were discarded. Similarly, several shorter contigs were identified in the primary contig-based assembly of both isolates even following the Dovetail scaffolding, and many of these contigs were fully redundant within the genome. Because the sequences were fully duplicated within larger chromosomes with nearly $100 \%$ sequence identity, these shorter contigs were considered assembly errors - perhaps due to the exceedingly high amount of raw input data at nearly 200x genome coverage - and were removed to produce the final number of contigs in the final assembly.

Still, there are some smaller contigs, especially within the 11-12 assembly, with a largely unknown identity or placement within the larger genome. A couple of these contigs that are less than $100 \mathrm{~kb}$ are the mitochondrial genome of M. phaseolina, including Contig 15 in the
11-12 assembly and Contigs 14, 16, 17, and 18 in the $\mathrm{Al}-1$ assembly. In the $\mathrm{Al}-1$ assembly, only Contig 15, a 41,517 bp contig, remains unplaced in the final assembly and does not contain any annotated transcripts based on annotation with MAKER. In contrast, the 11-12 assembly has 46 small contigs representing the nuclear genome remaining in the assembly, with 34 of them having at least one annotated gene and repeat. Unlike the smaller contigs of Al-1, the small contigs of the 11-12 genome had a greater number and diversity of transposable elements, including unique Penelope LINE transposons and ERV4 LTR transposons which may contribute to the difficulty of their scaffolding compared to Al-1. Chromosomal sized bands in pulsed field gel electrophoresis were observed that corresponded to the 0.87 and $1.24 \mathrm{Mb}$ contigs of the $\mathrm{Al}-1$ assembly (smaller bands were not seen); while a band corresponding to the 1.07 $\mathrm{Mb}$ contig of the 11-12 assembly was observed, a corresponding scaffold for a $0.79 \mathrm{Mb}$ band in the gel was not (Additional file 4: Figure S1). It is likely a number of the smaller contigs of 11-12 would be scaffolded to this $0.79 \mathrm{Mb}$ band. Additional methods of scaffolding, including the electronic mapping used by Nabsys (Providence, RI), could help to place some of these small contigs into larger scaffolds [30]. In Fusarium oxysporum it has been demonstrated the smaller chromosomes rich in repeats and transposons encode genes associated with pathogenesis [15]; it is unknown if is the case with the smaller contigs of the 11-12 isolate of the strawberry genotype which also displayed high numbers of transposons on the smaller contigs.

The functional annotation of these genomes was completed using RNA-Seq data derived from the 11-12 isolate grown in 10 different conditions to ensure that variation in gene expression was captured to obtain high-quality data for annotation. This extensive set of transcriptome data was used along with a representative set of protein sequences from taxonomically related fungi to create an updated and thorough functional annotation of the $M$ phaseolina genome. Despite using the most up-to-date available data and state-of-the-art computational tools to identify orthologous genes and predict gene function, functional annotations are still imperfect in that they use tools that base annotations upon proteins and domains that have been previously characterized in model organisms that are at best closely related to M. phaseolina and many times have only been fully characterized in different biological kingdoms. As a result, many predicted proteins are identified as a "gene of unknown function" and several of the clusters of orthologous genes determined through OrthoFinder or OrthoVenn2 don't have predicted functions or GO annotations. These tools are still useful in sorting the data that we have based on the annotation databases that are 
currently available, but more work needs to be done in the field of fungal functional genomics before these predictions can be more accurate and analysis like GO term enrichment can be more biologically meaningful.

When searching for genes associated with isolates representing the strawberry genotype, 10 genes from the 11-12 assembly were identified including enzymes, proteins involved in DNA binding, and several genes with unknown function. Despite a very rigorous pipeline to identify genes that were unique to isolates in the strawberry genotype, candidate genes were also present in isolates $14-26$ and $14-27$ that were initially recovered from strawberry but were not pathogenic in laboratory inoculations. Since a single, highly susceptible strawberry cultivar was evaluated in determining $M$. phaseolina pathogenicity; it is possible that these isolates may be pathogenic on other strawberry cultivars. Furthermore, these isolates were part of the less than $2 \%$ of the isolates recovered from strawberry that were not in the strawberry genotype. Testing with soil-based inoculum derived from an isolate within this small subgroup revealed it had a much lower level of virulence on strawberry when compared to isolates from the strawberry clade (B. Smith and F. Martin, unpublished).

Given these considerations, the list of candidate genes generated from an extensive presence-absence analysis of genes annotated in the strawberry isolate provides a strong foundation to evaluate potential causes of $M$. phaseolina pathogencity and virulence on a strawberry host. In some cases, genes with similar Pfam annotations have been shown to have significant roles in pathogenicity and host interactions. For example, genes M_11_ 12_v1_01448 and M11_12_v1_01460 are both likely transcription factors with Pfam domains including the Myb-like DNA binding domain and the $\mathrm{Zn}(2)$-Cys(6) binuclear cluster domain, respectively. Previous research has shown that transcription factors with the Myb-like DNA binding domain regulate the secretome in necrotrophic fungal pathogen [31] and a transcription factor with the $\mathrm{Zn}(2)$-Cys(6) binuclear cluster domain has a key role in regulating virulence in a fungus infecting wheat [32]. The cytochrome P450 gene, M11_12_v1_ 01466, is a member of a large group of genes that was found to be present in the $M$. phaseolina genome in high numbers and may be associated with secondary metabolites and overcoming host defenses [1]. A member of the Major Facilitator Superfamily, M11_12_v1_ 01458, is also part of a group of genes that was found in high numbers in the M. phaseolina genome [1] and has been shown to be important in mediating pathogenicity in other fungi [33]. These candidate genes, especially those with unknown functions, could have novel roles in pathogencity and virulence, but to fully test whether the candidate genes found predominantly in the strawberry genotype are responsible for conferring pathogenicity to M. phaseolina on strawberry, a knockout study using new technology like CRISPR would need to be done [34].

Beyond examining the presence or absence of particular genes in the genome, it is important to investigate the expression and interdependence of genes responsible for enabling an isolate of $M$. phaseolina to infect strawberry. Further studies examining gene expression or transcriptional networks during infection of strawberry by $M$. phaseolina may reveal a more complex role for some of these candidate genes. It will be interesting to see if additional genes or gene expression patterns unique to the strawberry genotype are observed once the smaller contigs in the 1112 assembly are joined into larger chromosome-sized contigs/scaffolds. The high quality genomes produced in this manuscript provides a valuable resource for future transcriptomic studies that will further elucidate which genes and pathways are involved in conferring host specificity to isolates of M. phaseolina infecting strawberry.

\section{Conclusions}

In summary, this work provides high-quality annotated genome assemblies for Macrophomina phaseolina isolates from strawberry and alfalfa hosts. These PacBiobased assemblies are an improvement over the previously published assembly for an isolate recovered from jute in that these assemblies have fewer contigs, longer contigs, and all the raw data from the sequencing and annotation is publicly available. Comparative genomics of these more complete assemblies has revealed largescale structural rearrangements in terms of chromosomal inversions and translocations. In addition, thirty additional isolates from strawberry and other hosts were sequenced with Illumina in this study, all of which provide valuable comparative genomics resources for this and other studies involving M. phaseolina. In this study, an analysis of gene presence/absence created a short list of candidate genes associated with isolates pathogenic on strawberry that may be involved in host preference to strawberry.

\section{Methods}

\section{M. phaseolina isolate growth and DNA extraction}

Cultures of M. phaseolina were recovered from infected plants collected in California. The 11-12 isolate was collected from strawberry in Santa Barbara County, CA, and the $\mathrm{Al}-1$ isolate was collected from alfalfa in Imperial County, CA. Tissue for DNA extraction was grown on potato dextrose broth (PDB) at $28{ }^{\circ} \mathrm{C}$ for two days. The cultures were then blended with sterile water and grown for another 2 days at $28^{\circ} \mathrm{C}$ to produce a hyphal mat. This tissue was strained, rinsed with sterile water, blotted dry, and flash frozen. The tissue was lyophilized and DNA was extracted using the Blood and Cell 
Culture kit (Qiagen, Redwood City, CA) with the modifications described in the Qiagen user-developed protocol for the isolation of genomic DNA from plants and filamentous fungi using the Qiagen Genomic tip. The only exception was that buffer G2 in the kit was used as the lysis buffer. The quality of the DNA was tested by running the samples on a $0.8 \%$ agarose gel and the concentration measured using a Qubit (Thermo Fisher Scientific, Waltham, MA). The samples were submitted for PacBio RSII sequencing to the DNA Technologies Core at the Genome Center of the University of California (Davis, CA). Fourteen SMRT cells were sequenced for the isolate representing the main strawberry genotype (11-12; SAMN08294525) and 10 SMRT cells were sequenced for an isolate recovered from alfalfa representing another genotype (Al-1; SAMN08448485). The DNA was also submitted to Michigan State University Research Technology Support Facility Genomics Core (East Lansing, MI) for mate pair library preparation and Illumina paired-end sequencing. The mate pair libraries were prepared using the Illumina Nextera Mate Pair Library Preparation Kit. The inserts for the mate pair libraries were prepared by fragmenting the DNA sample and running it on a polyacrylamide gel to separate the fragment sizes. The samples were run on an Agilent Bioanalyzer DNA 12000 chip which showed that the mate pair insert fragment ranges for isolate 11-12 were 3.5, 5.0, and $7.0 \mathrm{~kb}$ for expected 3,6 , and $9 \mathrm{~kb}$ insert libraries, respectively. These libraries were sequenced using Illumina HiSeq 2500 High Output flow cell (v4) using $125 \mathrm{bp}$ paired-end sequencing with the HiSeq SBS reagents. For the Al-1 sample, mate pair inserts of 3.1, 5.7 , and $8.5 \mathrm{~kb}$ were created by fragmentation and were sequenced on an Illumina MiSeq v2 flow cell to provide $150 \mathrm{bp}$ paired-end data. DNA for these two isolates was also sent to the USDA-ARS Genomics and Bioinformatics Research Unit (Stoneville, MS) for a single MiSeq run using the same library preparation techniques noted above to provide $300 \mathrm{bp}$ paired-end data. The paired-end and mate pair data for 11-12 and $\mathrm{Al}-1$ is available on NCBI under accession number SAMN08294525 and SAMN08448485, respectively.

Additional sequencing was completed using DNA extracted from lyophilized tissue of 30 isolates of $M$. phaseolina using the Qiagen DNeasy Plant Mini Kit following the manufacturer's directions for lyophilized plant tissue except the tissue lysis step was increased to one hour. This DNA was submitted to either Michigan State University Research Technology Support Facility Genomics Core or DNA Technologies Core at the Genome Center of the University of California for $150 \mathrm{bp}$ paired-end sequencing using the Illumina HiSeq4000. The data can be located at NCBI under accession numbers SAMN09764508-SAMN09764537 (for a full list of isolates and the corresponding accession numbers see Table 4).

\section{M. phaseolina isolate growth and RNA extraction}

Isolate 11-12 of $M$. phaseolina was grown under a variety of conditions to express a wide range of genes for RNA sequencing to be used for genome annotation. All the samples were started on PDB and were grown at $28^{\circ} \mathrm{C}$ for two days. Three samples were blended with water and were transferred to $15^{\circ} \mathrm{C}, 35^{\circ} \mathrm{C}$, or 24 -h light and were grown for an additional two days. One sample was grown at $28^{\circ} \mathrm{C}$ for 7 days after it was blended. Other cultures that were started on PDB were transferred to a different media by removing the growing culture with a rubber policeman and rinsing it briefly with distilled water before blending it with a new media and transferring it to a new set of petri plates to grow for 2 days at $28^{\circ} \mathrm{C}$. The other media used included PDB with 2 and $4 \% \mathrm{NaCl}$ and PDB adjusted to a $\mathrm{pH}$ of 3.5 or 8 with $\mathrm{HCl}$ or $\mathrm{NaOH}$. Additional media included $100 \mathrm{mM}$ ammonium sulfate and sterilized macerated strawberry crown extract. All the fungal tissue was collected from the various growth conditions by straining the media from the tissue, rinsing the tissue with sterile water several times, and blotting the tissue dry. The tissue mat was then rolled into a cylinder and flash-frozen in liquid nitrogen and stored at $-80^{\circ} \mathrm{C}$ until the RNA was extracted.

RNA was extracted using the Qiagen RNeasy Plant Mini Kit from flash-frozen tissue grown in each of the conditions described above. The RNA was submitted to the Michigan State University Research Technology Support Facility Genomics Core and was prepared using the Illumina TruSeq Stranded mRNA Library prep kit and was sequenced using $125 \mathrm{bp}$ paired-end reads from the Illumina HiSeq 2500. The reads were submitted to NCBI (SAMN09699088, SAMN09699089, SAMN09699090, SAMN09699091, SAMN09699092, SAMN09699093, SAMN09699094, SAMN09699095, SAMN09699096, SAMN09699097).

\section{Genome assembly with FALCON}

The genomes of $M$. phaseolina isolates 11-12 and Al-1 were assembled from the PacBio data using the FALCON assembly pipeline v. 0.3.0 [29]. First, the reads were converted from the raw bax.h5 format to subreads.fasta using the pbh5tools v. 0.8.0. FALCON was run for isolate 11-12 using the following parameter options: input_type $=$ raw, length_cutoff $=10$, 000 , length_cutoff_pre $=10,000$, pa_HPCdaligner_option $=$ -v -B128 -t16 -e.70 -11000 -s400 -T4, ovlp_HPCdaligner_op-

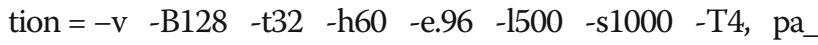
DBsplit_option and ovlp_DBsplit_option $=-\times 500-$ s100, fal con_sense_option $=-$-output_multi - -min_idt $0.70 \quad-$-min cov 4 --max_n_read 200 --n_core 6, and overlap_filtering setting = --max_diff 300 --max_cov 500 --min_cov 5 --bestn 10 --n_core 15. FALCON was run for isolate $\mathrm{Al}-1$ using the same parameters except for the following changes: length cutoff $=15,000$, length_cutoff_pr $=11,000$, and overlap_ 
filtering_setting = --max_diff 100 --max_cov 450 --min_cov 10 --bestn 10 --n_core 15 . The resulting p_ctg.fa output file was used as the input genome assembly for subsequent analyses.

\section{Genome assembly improvement with PILON}

The mate pair and paired-end reads used to polish the FALCON-based assemblies were first trimmed using NxTrim v. 0.4.1 [35] using the -justmp and -separate options and or Trimmomatic v. 0.36 [36]. Bowtie2 (v 2.2.6) was used to map the trimmed mate pair and paired-end reads to the FALCON-based assemblies of each genome [37]. Bowtie2 was run for isolate 11-12 data using the -very-sensitive option and with the -I parameter set at 2000,3500 , or 4500 for a minimum fragment length and the -X parameter set at 5000, 6500, or 9000 for a maximum fragment length for the $\sim 3.4 \mathrm{~kb}$, $6 \mathrm{~kb}$, and $9 \mathrm{~kb}$ mate pair insert libraries, respectively. Bowtie2 was run using the -very-sensitive option with the -I parameter set at 1500,4200 , or 6500 and the $-\mathrm{X}$ parameter set at 4500,7200 , or 10,500 , respectively for the mate pair libraries that were approximately $3 \mathrm{~kb}, 6$ $\mathrm{kb}$, and $9 \mathrm{~kb}$ for isolate Al-1 data. The mapped reads were converted to sorted bam files using SAMTools v. $1.17[38,39]$ and were used as -jumps (mate-pair) or frags (paired-end) input data for PILON v. 1.16 [19] to polish the FALCON-based assemblies. The corrected fasta file from PILON that had SNPs, indels, gaps, and local misassemblies fixed was used as the reference sequence for a second round of paired-end and matepair read mapping using the parameters for Bowtie2 described above. The sorted bam files from the second round of Bowtie2 mapping were used for a second PILON run using the same parameters described above. The final assembly from each second round of PILONbased polishing was used as the input assembly for $\mathrm{Hi}-\mathrm{C}$ analysis by Dovetail (Santa Cruz, CA).

\section{Hi-C scaffolding}

Hyphal mats of M. phaseolina from isolates 11-12 and Al-1 were collected during the log phase of growth in $\mathrm{PDB}$, rinsed, and partially dried on filter paper by pulling a low vacuum. The tissue was flash-frozen in liquid nitrogen and sent to Dovetail Genomics (Santa Cruz, $\mathrm{CA}$ ). The assembly was scaffolded following $\mathrm{Hi}-\mathrm{C}$ analysis using the HiRise method and the gaps in the scaffolds were filled in with PBJelly using the previously described PacBio subreads. The contigs from the Dovetail gap-filled assembly were analyzed using CLC Genomics Workbench v. 9.5.3. The Align Contigs tool in the Genome Finishing Module was used to align the contigs to themselves using BLAST [40] with a word size of 20, a maximum e-value of 0.01 , and minimum match size of 100. Contigs were eliminated from the final assembly that were more than $99.5 \%$ contained within another contig and had greater than $99.5 \%$ identity to that contig. Contigs less than $1000 \mathrm{bp}$ were also removed. The contigs/scaffolds were reordered from longest to shortest and renamed so that the longest contig/scaffold was named Contig 1 and the shortest contig/scaffold was Contig $\mathrm{n}$, where $\mathrm{n}$ equals the number of total contigs/ scaffolds in the assembly. The final assemblies for isolates 11-12 (PRJNA428521) and Al-1 (PRJNA432410) are available on NCBI. Assemblies were evaluated for quality and completeness using QUAST v. 4.3 [41] and BUSCO2 v. 2.0 [42] with both the eukaryotic and fungal dataset.

\section{Genome annotation}

MAKER [43] was used to structurally annotate both genomes, using RNA-Seq reads produced from 11-12 and predicted protein sequences from the NCBI GenBank assemblies of closely-related fungal species (Fusarium oxysporum GCA_00149955, Macrophomina phaseolina MS6 GCA_000302655, Penicillium chrysogenum GCA 000710275, Grosmannia clavigera GCA_000143105, Aspergillus nidulans GCA_000149205, Trichoderma reesi GCA 000167675, and Podospora anserina GCA_000226545). Trimmomatic v. 0.36 was used to trim the RNA-Seq reads using the default settings with the minimum read length set at 50 . The trimmed paired reads were then mapped to each genome using STAR aligner v. 2.3.0e [44]. SAMTools v.0.1.19 was used to convert the aligned sam file into a sorted bam file. Trinity v. 2.4.0 [45] was used to create gene models using the -genome-guided_bam option, a maximum intron length of 5000, the jaccard clipping option, and the SS_lib_ type RF. The gene models produced by Trinity from each RNA-Seq data set were used as EST evidence when running MAKER v. r1228. Repeat masking was done within MAKER RepeatMasker [22] using the RepBase [46] for all model organisms with soft masking. The specific transposable elements were curated from the output of RepeatMasker for each genome. De novo gene predictions were made using SNAP [47], Augustus [48] and Genemark v. 4.32 [49]. Specific MAKER options were set so that the AED_threshold was 1 , the pred_flank was 200, the split_hit was 10,000, and the single_length was 250. A MAKER standard gene set was made which included genes that had evidence and or Pfam support with a cutoff of $1 \mathrm{e}-10$ and encoded a protein of at least 50 amino acids. The transcripts file, protein sequences, gff files, and final assemblies are available at Data Dryad.

\section{Functional annotation}

The MAKER standard gene set was subject to gene functional annotation using Trinotate v3 [50]. Both protein and transcript sequences were queried against the Swiss-Prot database [51] using BLASTP and BLASTX [40], respectively. In addition, protein sequences were 
scanned for conserved Pfam domains in the Pfam-A database [52] using HMMER v3 [53]. Both SignalP [54] and TMHMM [55] were used to identify signal peptide and transmembrane domains in the predicted protein sequences. Outputs from BLASTX, BLASTP, HMMER, SignalP, and TMHMM were used to generate the final functional annotation report. To characterize the carbohydrateactive enzymes in the fungal genome, protein sequences were searched against the dbCAN HMMdb release v7 [56], and the identified carbohydrate enzymes were assigned to appropriate CAZy enzyme classes (GHs, GTs, PLs, CEs, CBMs, and AAs).

\section{Assembly and sequencing of other isolates of $M$. phaseolina}

Illumina sequencing data from the additional 30 isolates of $M$. phaseolina were analyzed using CLC Workbench (Table 4). The reads were trimmed using the Illumina TruSeq adapter set with a quality trim setting at 0.05 and a minimum read length of $30 \mathrm{bp}$. These trimmed reads were assembled into draft assemblies using CLC Workbench using the de novo assembly tool with an automatic bubble size and with a word size that was manually adjusted from 25 to 50 to provide the assembly with the best N50. The assemblies were used to create BLAST databases for downstream analyses within CLC Workbench and were also exported for downstream analyses using other programs. The statistics for the jute isolate MphMS6_1.0 in Table 1 were generated by using the publicly available assembly, GCA_000302655.1, which is assembled at the contig and not the scaffold level.

\section{Genetic structural variation}

To evaluate genome structural rearrangement, $M$. phaseolina files were prepared for Assemblytics [57] using MUMmer v. 3.23 [58] with the following settings: -1 100, $-c 500$. The output file from MUMmer was used as the input for Assemblytics using the unique sequence length of 10,000 , a maximum variant size of 10,000 , and a minimum variant size of 1 . A progressive MAUVE alignment using MAUVE v. 2.3.1 [59] was also run with the final 11-12 assembly as the reference sequence to which the final $\mathrm{Al}-1$ assembly was aligned.

\section{Functional comparative genomics}

Orthologs between $M$. phaseolina from strawberry and other isolates of $M$. phaseolina as well as 17 other closelyrelated fungal species were identified using OrthoFinder v. 1.1.3 [60]. The fungal taxonomy and protein sequences used in this analysis can be found in Additional file 1: Table S1. OrthoVenn2 was used to create plots and to do additional GO term enrichment analyses of specific groups [61]. All enriched GO term groups had an E-value of 0.01 and an inflation value of 1.5 as specified with the OrthoVenn2 online toolkit.

\section{Identification of genes unique to the main strawberry genotype}

Illumina-based paired-end DNA sequencing was analyzed for 12 isolates not pathogenic on strawberry and 18 isolates pathogenic on this host to identify genes that are unique to $M$. phaseolina isolates from the strawberry genotype. These reads were trimmed using Trimmomatic and were mapped to the 11-12 genome using STAR [44] with the -outFilterMultimapNmax set to 1 and the -outFilterMismatchNmax set to 0 so that only the reads that were perfectly mapped to the genome were included in the output sam file. The file was then converted into a sorted bam file that was used as an input for HTSeq-count from HTSeq v. 0.6.1p1 [62]. HTSeq-count was used with the $-\mathrm{f}$ bam, $-\mathrm{s}$ no and $-\mathrm{t}$ gene parameters using the gff3 file from 11-12 as the gene model input. The read counts per gene from each of the isolates was analyzed using the DESeq package $\mathrm{v}$. 1.32.0 [63] in $R$ v. 3.5.0. Each set of read counts were normalized according to the library size and the counts for each gene for each isolate were exported to Excel. Excel was used to sort the count data files to identify genes that had no reads mapping from the nonpathogenic isolates and had several reads mapping from the pathogenic isolates. These genes were considered as candidate genes unique to the strawberry genotype to be used for downstream analyses. Additional analysis of candidates was done using the BLAST functions in CLC Workbench in which the 11-12 candidate gene sequences were used as queries to BLAST against a local database created in CLC Workbench from the assembly of each isolate. The top BLAST hit from each assembly was considered as the homolog for the candidate gene in that isolate and was used to manually check for the presence of indels or SNPs compared to the gene model from the 11-12 isolate using sequence alignments in CLC Workbench.

\section{Pathogenicity testing}

M. phaseolina isolates were tested for pathogenicity on strawberry by growing cultures on potato dextrose agar (PDA) at $25^{\circ} \mathrm{C}$ and allowing the isolates to colonize sterile toothpicks placed on top of the growing cultures for 10 days. The colonized toothpicks were used as the inoculum source. Prior to inoculation, strawberry plants of cultivar (cv.) Albion were grown in the greenhouse from crowns for 28 days. Plants were inoculated by making a $6 \mathrm{~mm}$ deep hole in each crown with a metal probe and then placing the colonized toothpick inside the hole. Sterile toothpicks were placed in each crown as a negative control and toothpicks colonized with isolate 11-12 
were used as a positive control. For each trial, 8 plants were inoculated per treatment and were placed in a growth chamber set at $30^{\circ} \mathrm{C}$ and were watered as needed. The plants were rated from 1 to 4 weeks post inoculation using a 1-3 scale in which a rating of 1 was a symptomless plant, a rating of 2 indicated a moderate amount of wilt and die back, and a rating of 3 indicated complete collapse and death of the plant. The final ratings were analyzed using the nonparametric Kruskal-Wallis test in R followed by pairwise comparisons using the Wilcoxon rank sum test; isolates were considered to be pathogenic if the adjusted $P$ value compared to the negative control was < 0.05 [64] (Table 4). Each isolate was tested in 1-3 separate trials.

\section{Supplementary information}

Supplementary information accompanies this paper at https://doi.org/10. 1186/s12864-019-6168-1.

Additional file 1: Table S1. List of all of the contig lengths for the 1112 and Al-1 Macrophomina phaseolina genome assemblies.

Additional file 2: Table S2. List of fungal species used in OrthoMCL analysis.

Additional file 3: Table S3. Percentage of shared orthologous genes for 20 species of fungi closely related to and including three isolates of Macrophomina phaseolina. Data corresponds to Fig. 3.

Additional file 4: Figure S1. Pulsed-field electrophoresis gel of Macrophomina phaseolina isolates 11-12 and Al-1 run on a BioRad CHEF DR-II in a $0.8 \%$ BioRad certified megabase agarose gel with a ramping interval of $100-180 \mathrm{~min}$ for $65 \mathrm{~h}$ at $4 \mathrm{v} / \mathrm{cm}$ run at a constant $4{ }^{\circ} \mathrm{C}$ temperature. Size markers in the left lanes are Hansenula wingei and Saccharomyces cerevisiae.

\section{Abbreviations}

11-12: Macrophomina phaseolina isolated from strawberry in 2011; Al1: Macrophomina phaseolina isolated from alfalfa; BLAST: Basic Local Alignment Search Tool; BUSCO: Benchmarking Universal Single Copy Orthologs; CAZY: Carbohydrate-Active enzymes; CRISPR: Clustered Regularly Interspaced Short Palindromic Repeats; GO: Gene Ontology; NCBI: National Center for Biotechnology Information; PacBio: Pacific Biosciences; PDA: Potato dextrose agar; PDB: Potato dextrose broth; QUAST: Quality Assessment Tool for Genome Assemblies; SMRT: Single Molecule Real-Time; SNP: Single nucleotide polymorphism

\section{Acknowledgements}

We thank Steve Koike formerly at the University of California Cooperative Extension for assistance in collecting and providing various samples of Macrophomina phaseolina. We thank John Johnston at Michigan State University for maintaining servers and uploading programs. We thank Brett Smith and Sam Cude for assistance in conducting pathogenicity tests and Brian Scheffler of the USDA-ARS Genomics and Bioinformatics Research Unit in Stoneville, MS for the MiSeq data for isolates 11-12 and Al-1.

\section{Authors' contributions}

AKB designed the experiments, conducted that data analysis, and wrote the manuscript. KLC contributed to experimental design, provided scripts for genome annotation, and provided the resources and technical support for all the bioinformatic data analysis. JW conducted the functional annotation. MLR extracted DNA for PacBio sequencing and conducted initial population studies. FNM worked on initial genome assemblies, provided guidance for experimental design and data analysis and obtained the funding to conduct this work. All authors have read, edited, and approved the final manuscript.

\section{Funding}

The authors gratefully acknowledge funding from the California Department of Food and Agriculture's Specialty Crop Block Grant Program grant SCB14052 and the California Strawberry Commission that supported this work. These funding bodies played no role in the design of the study and collection, analysis, interpretation of data and in writing the manuscript.

\section{Availability of data and materials}

Digital storage of fasta and .gff files for the genome can be found at Data Dryad: https:/doi.org/10.5061/dryad.zkh18935h.

The datasets described in this manuscript are available through NCBI. Illumina sequencing of 30 isolates of $M$. phaseolina - BioProject PRJNA484400 RNA sequencing of 11-12 libraries - BioProject PRJNA428521.

Sequencing and genome assembly for Al-1 isolate - BioProject PRJNA432410.

Sequencing and genome assembly for 11-12 isolate - BioProject PRJNA428521.

Ethics approval and consent to participate

Not applicable

\section{Consent for publication}

Not applicable

\section{Competing interests}

The authors declare that they have no competing interests.

Received: 27 March 2019 Accepted: 3 October 2019

Published online: 04 November 2019

\section{References}

1. Islam MS, Haque MS, Islam MM, Emdad EM, Halim A, Md Q, Hossen M, Hossain MZ, Ahmed B, Rahim S, Rahman MS, Alam MM, Hou S, Wan X, Saito JA, Alam M. Tools to kill: genome of one of the most destructive plant pathogenic fungi Macrophomina phaseolina. BMC Genomics. 2012;13:493.

2. Gupta GK, Sharma SK, Rameke R. Biology, epidemiology and management of the pathogenic fungus Macrophomina phaseolina (Tassi) Goid with special reference to charcol rot of soybean. J Phytopathol. 2012;160:167-80.

3. Kaur S, Dhillon GS, Brar SK, Vallad GE, Chand R, Chauhan VB. Emerging phytopathogen Macrophomina phaseolina : biology, economic importance and current diagnostic trends. Crit Rev Microbiol. 2012;38(2):136-51.

4. Zveibil A, Mor N, Gnayem N, Freeman S. Survival, host-pathogen interaction, and management of Macrophomina phaseolina on strawberry in Israel. Plant Dis. 2012;96(2):265-72.

5. Mihail JD. Macrophomina phaseolina: Spatio-temporal dynamics of inoculum and of disease in a highly susceptible crop. Phytopathology. 1989;79:848-55.

6. Khan AN, Shair F, Malik K, Hayat Z, Khan MA, Hafeez FY, Hassan MN Molecular identification and genetic characterization of Macrophomina phaseolina strains causing pathogenicity on sunflower and chickpea. Front Microbiol. 2017:8:1309.

7. Su G, Suh R, Schneider W, Russin JS. Host specialization in the charcol rot fungus, Macrophomina phaseolina. Phytopathology. 2001;91(2):120-6.

8. Koike ST, Arias RS, Hogan CS, Martin FN, Gordon TR. Status of Macrophomina phaseolina on strawberry in California and preliminary characterization of the pathogen. Int J Fruit Sci. 2016;16:148-59.

9. Mertely J, Seijo T, Peres N. First report of Macrophomina phaseolina causing a crown rot of strawberry in Florida. Plant Dis. 2005;89:434.

10. Koike ST. Crown rot of strawberry caused by Macrophomina phaseolina in California. Plant Dis. 2008;92(8):1253.

11. National Agricultural Statistics Services. Noncitrus fruits and nuts 2018 summary. United States Department of Agricutlure. June 2019. ISSN:19482698. https://www.nass.usda.gov/Publications/Todays_Reports/reports/ ncit0619.pdf.

12. Arias RS, Ray JD, Mengistu A, Scheffler BE. Discriminating microsatellites from Macrophomina phaseolina and their potential association to biological functions. Plant Pathol. 2011;60(4):709-18.

13. Faino L, Seidl MF, Datema E, Van Den Berg GCM, Janssen A, Wittenberg $A H J$, Thomma BPHJ. Single-molecule real-time sequencing combined with optical mapping yields completely finished fungal genome. MBio. 2015;6(4):e00936-15. 
14. Faino L, Farmer AD, Papasotiriou DG, Zhou S, Seidl MF, Cottam E, Edel D, Hahn M, Schwartz DC, Dietrich RA, Widdison S, Scalliet G. A gapless genome sequence of the fungus Botrytis cinerea. Mol Plant Path. 2017;18(1):75-89.

15. Van Dam P, Fokkens L, Ayukawa Y, Van Der Gragt M, Ter Horst A, Brankovics B, Houterman PM, Arie T, Rep M. A mobile pathogenicity chromosome in Fusarium oxysporum for infection of multiple cucurbit species. Sci Rep. 2017;7:9042.

16. Bao J, Chen M, Zhong Z, Tang W, Lin L, Zhang X, Jiang H, Zhang D, Miao C, Tang H, Zhang J, Lu G, Ming R, Norvienyeku J, Wang B, Wang Z. PacBio sequencing reveals transposable elements as a key contributor to genomic plasticity and virulence variation in Magnaporthe oryzae. Mol Plant. 2017; 10(11):1465-8.

17. Derbyshire M, Denton-Giles M, Hegedus D, Seifbarghi S, Rollins J, Van Kan J, Seidl MF, Faino L, Mbengue M, Navaud O, Raffaele S, Hammond-Kosack K, Heard S, Oliver R. The complete genome sequence of the phytopathogenic fungus Sclerotinia sclerotiorum reveals insights into the genome architecture of broad host range pathogens. Genome Biol Evol. 2017;9(3):593-618.

18. Dallery JF, Lapalu N, Zampounis A, Pigné S, Luyten I, Amselem J, Wittenberg AHJ, Zhou S, de Queiroz MV, Robin GP, Auger A, Hainaut M, Henrissat B, Kim KT, Lee YH, Lespinet O, Schwartz DC, Thon MR, O'Connell RJ. Gapless genome assembly of Colletotrichum higginsianum reveals chromosome structure and association of transposable elements with secondary metabolite gene clusters. BMC Genomics. 2017;18:667.

19. Walker BJ, Abeel T, Shea T, Priest M, Abouelliel A, Sakthikumar S, Cuomo CA, Zeng Q, Wortman J, Young SK, Earl AM. Pilon: an integrated tool for comprehensive microbial variant detection and genome assembly improvement. PLoS One. 2014;9(11):e112963.

20. Putnam NH, Connell BO, Stites JC, Rice BJ, Hartley PD, Sugnet CW, Haussler D, Rokhsar DS. Chromosome-scale shotgun assembly using an in vitro method for long-range linkage. Genome Res. 2016;26:342-50.

21. Teh BT, Lim K, Yong CH, Ng CCY, Rao SR, Rajasegaran V, Lim WK, Ong CK, Chan K, Cheng VKY, Soh PS, Swarup S, Rozen SG, Nagarajan N, Tan P. The draft genome of tropical fruit durian (Durio zibethinus). Nat Genet. 2017; 49(11):1633-41.

22. Smit AFA, Hubley R, Green, P. RepeatMasker Open-4.0. http://www. repeatmasker.org.

23. Arkhipova IR. Distribution and phylogeny of penelope-like elements in eukaryotes. Syst Biol. 2006:55(6):875-85.

24. Castanera R, López-Varas L, Borgognone A, LaButti K, Lapidus A, Schmutz J, Grimwood J, Pérez G, Pisabarro AG, Grigoriev IV, Stajich JE, Ramírez L. Transposable elements versus the fungal genome: impact on whole-genome architecture and transcriptional profiles. PLoS Genet. 2016;12(6):e1006108.

25. Wolters PJ, Faino L, van den Bosch TBM, Evenhuis B, Visser RGF, Seidl MF, Vleeshouwers VGAA. Gapless genome assembly of the potato and tomato early blight pathogen Alternaria solani. Mol Plant-Microbe Interact. 2018; 31(7):692-4

26. Proctor RH, McCormick SP, Alexander NJ, Desjardins AE. Evidence that a secondary metabolic biosynthetic gene cluster has grown by gene relocation during evolution of the filamentous fungus Fusarium. Mol Micro. 2009;74(5):1128-42.

27. Khan AN, Shari F, Malik K, Hayatt Z, Khan MA, Hafeez FY, Hassan MN. Molecular identification and genetic characterization of Macrophomina phaseolina strains causing pathogencity on sunflower and chickpea. Front Microbiol. 2017:8:1309.

28. Burkhardt A, Ramon ML, Smith B, Koike ST, Martin M. Development of molecular methods to detect Macrophomina phaseolina from strawberry plants and soil. Phytopath. 2018;12:1386-94.

29. Chin CS, Peluso P, Sedlazeck FJ, Nattestad M, Concepcion GT, Clum A, Dunn C, O'Malley R, Figueroa-Balderas R, Morales-Cruz A, Cramer GR, Delledonne M, Luo C, Ecker JR, Cantu D, Rank DR, Schatz MC. Phased diploid genome assembly with single-molecule real-time sequencing. Nat Methods. 2016; 13(12):1050-4

30. Kaiser MD, Davis JR, Grinberg BS, Oliver JS, Sage JM, Seward L, Bready B. Automated structural variant verification in human genomes using singlemolecule electronic DNA mapping. bioRxiv. 2017. https://doi.org/10.1101/ 140699. Accessed 5 Dec 2018.

31. Verma S, Gazara RK, Verma PK. Transcription factor repertoire of necrotrophic fungal phytopathogen Ascochyta rabiei: predominance of MYB transcription factors as potential regulators of secretome. Front Plant Sci. 2017;8:1037.
32. Rybak K, See PT, Phan HTT, Syme RA, Moffat CS, Oliver RP, Tan K-C. A functionally conserved $\mathrm{Zn}_{2} \mathrm{Cys}_{6}$ binuclear cluster transcription factor class regulates necrotrophic effector gene expression and host-specific virulence of two major Pleosporales fungal pathogens of wheat. Mol Plant Pathol. 2017;18(3):420-34

33. Vela-Corcia D, Srivastava DA, Dafa-Berger A, Roten N, Barda O, Levy M. MFS transporter from Botrytis cinerea provides tolerance to glucosinolate-breakdown products and is required for pathogenicity. Nature Com. 2019;10:2886.

34. Shi TQ, Liu GN, Ji RY, Shi K, Song P, Ren LJ, Huang H, Ji XJ. CRISPR/Cas9based genome editing of the filamentous fungi: the state of the art. Appl Microbiol Biotechnol. 2017;101:7435-43.

35. O'Connell J, Schulz-Trieglaff O, Carlson E, Hims MM, Gormley NA, Cox AJ. NxTrim: optimized trimming of Illumina mate pair reads. Bioinformatics. 2015;31(12):2035-7.

36. Bolger AM, Lohse M, Usadel B. Trimmomatic: a flexible trimmer for Illumina sequence data. Bioinformatics. 2014;30(15):2114-20.

37. Langmead B, Salzberg SL. Fast gapped-read alignment with bowtie 2. Nat Methods. 2012;9(4):357.

38. Li H, Handsaker B, Wysoker A, Fennell T, Ruan J, Homer N, Marth G, Abecasis $\mathrm{G}$, Durbin R. The sequence alignment/map format and SAMtools. Bioinformatics. 2009;25(16):2078-9.

39. Li H. A statistical framework for SNP calling, mutation discovery, association mapping and population genetical parameter estimation from sequencing data. Bioinformatics. 2011;27(21):2987-93.

40. Altschul SF, Gish W, Miller W, Myers EW, Lipman DJ. Basic local alignment search tool. J Mol Biol. 1990;215(3):403-10.

41. Gurevich A, Saveliev V, Vyahhi N, Tesler G. QUAST: quality assessment tool for genome assemblies. Bioinformatics. 2013;29(8):1072-5.

42. Simão FA, Waterhouse RM, loannidis P, Kriventseva EV, Zdobnov EM. BUSCO: assessing genome assembly and annotation completeness with single-copy orthologs. Bioinformatics. 2015;31(19):3210-2.

43. Cantarel BL, Korf I, Robb SMC, Parra G, Ross E, Moore B, Holt C, Alvarado AS, Yandell M. MAKER: an easy-to-use annotation pipeline designed for emerging model organism genomes. Genome Res. 2008;18:188-96.

44. Dobin A, Davis CA, Schlesinger F, Drenkow J, Zaleski C, Jha S, Batut P, Chaisson M, Gingeras TR. STAR: ultrafast universal RNA-seq aligner. Bioinformatics. 2013;29(1):15-21.

45. Grabherr MG, Haas BJ, Yassour M, Levin JZ, Thompson DA, Amit I, Adiconis X, Fan L, Raychowdhury R, Zeng Q, Chen Z, Mauceli E, Hacohen N, Gnirke A, Rhind N, Di Palma F, Birren BW, Nusbaum C, Lindblad-Toh K, Friedman N, Regev A. Full-length transcriptome assembly from RNA-Seq data without a reference genome. Nat Biotechnol. 2011;29(7):644-52.

46. Bao W, Kojima KK, Kohany O. Repbase update, a database of repetitive elements in eukaryotic genomes. Mob DNA. 2015;6:11.

47. Korf I. Gene finding in novel genomes. BMC Bioinformatics. 2004;5:59.

48. Ter-hovhannisyan V, Lomsadze A, Chernoff YO, Borodovsky M. Gene prediction in novel fungal genomes using an $a b$ initio algorithm with unsupervised training gene prediction in novel fungal genomes using an $a b$ initio algorithm with unsupervised training. Genome Res. 2008; 18(12):1979-90.

49. Borodovsky M, Mclninch J. GeneMark: parallel gene recognition for both strands. Comput Chem. 1993;17(2):123-33.

50. Bryant DM, Johnson K, DiTommaso T, Tickle T, Couger MB, Payzin-Dogru D, Lee TJ, Leigh ND, Kuo T-H, Davis FG, Bateman J, Bryant S, Guzikowski AR, Tsai SL, Coyne S, Ye WW, Freeman RM, Peshkin L, Tabin CJ, Regev A, Haas BJ, Whited JL. A tissue-mapped axolotl de novo transcriptome enables identification of limb regeneration factors. Cell Rep. 2017;18(3):762-76.

51. Apweiler R, Bairoch A, Wu CH, Barker WC, Boeckmann B, Ferro S, Gasteiger E, Huang H, Lopez R, Magrane M, Martin MJ, Natale DA, O'Donovan C Redaschi N, Yeh LS. UniProt: the universal protein knowledgebase. Nucleic Acids Res. 2004;32:115D-19.

52. Finn RD, Coggill P, Eberhardt RY, Eddy SR, Mistry J, Mitchell AL, Potter SC, Punta M, Qureshi M, Sangrador-Vegas A, Salazar GA, Tate J, Bateman A. The Pfam protein families database: towards a more sustainable future. Nucleic Acids Res. 2016;44:D279-85.

53. Mistry J, Finn RD, Eddy SR, Bateman A, Punta M. Challenges in homology search: HMMER3 and convergent evolution of coiled-coil regions. Nucleic Acids Res. 2013;41(12):e121.

54. Petersen TN, Brunak S, von Heijne G, Nielsen H. SignalP 4.0: discriminating signal peptides from transmembrane regions. Nat Methods. 2011;8:785. 
55. Möller S, Croning MDR, Apweiler R. Evaluation of methods for the prediction of membrane spanning regions. Bioinformatics. 2001;17(7):646-53.

56. Zhang $H$, Yohe T, Huang L, Entwistle S, Wu P, Yang Z, Busk PK, Xu Y, Yin Y. DbCAN2: a meta server for automated carbohydrate-active enzyme annotation. Nucleic Acids Res. 2018;46:W95-W101.

57. Nattestad M, Schatz MC. Assemblytics: a web analytics tool for the detection of assembly-based variants. Bioinformatics. 2016;32(19):3021-3.

58. Kurtz S, Phillippy A, Delcher AL, Smoot M, Shumway M, Antonescu C, Salzberg SL. Versatile and open software for comparing large genomes. Genome Biol. 2004;5:R12.

59. Darling AE, Mau B, Perna NT. progressiveMauve: multiple genome alignment with gene gain, loss and rearrangement. PLoS One. 2010; 5(6):e11147.

60. Emms DM, Kelly S. OrthoFinder: solving fundamental biases in whole genome comparisons dramatically improves orthogroup inference accuracy. Genome Biol. 2015;16:157.

61. Xu L, Dong Z, Fang L, Luo Y, Wei Z, Guo H, Coleman-Derr D, Xia Q Wang Y. OrthoVenn2: a web server for whole-genome comparison and annotation of orthologous clusters across multiple species. Nucleic Acids Res. 2019;47:W52-8.

62. Anders S, Pyl PT, Huber W. HTSeq - a Python framework to work with highthroughput sequencing data. Bioinformatics. 2015;31(2):166-9.

63. Anders $\mathrm{S}$, Huber W. Differential expression analysis for sequence count data. Genome Biol. 2010;11:R106.

64. Benjamini Y, Hochberg Y. Controlling the false discovery rate: a practical and powerful approach to multiple testing. J Royal Statistical Society Series B. 1995;57(1):289-300.

\section{Publisher's Note}

Springer Nature remains neutral with regard to jurisdictional claims in published maps and institutional affiliations.

\section{Ready to submit your research? Choose BMC and benefit from:}

- fast, convenient online submission

- thorough peer review by experienced researchers in your field

- rapid publication on acceptance

- support for research data, including large and complex data types

- gold Open Access which fosters wider collaboration and increased citations

- maximum visibility for your research: over $100 \mathrm{M}$ website views per year

At BMC, research is always in progress.

Learn more biomedcentral.com/submissions 